\title{
Stability Analysis of a Magneto Micropolar Fluid Layer by Variational Method
}

\author{
Joginder Singh Dhiman ${ }^{1}$, Gurdeep Singh ${ }^{2, *}$ \\ ${ }^{1}$ Department of Mathematics, Himachal Pradesh University, Shimla 171005, India \\ ${ }^{2}$ Department of Mathematics, GSSS Ghanari Distt Una, Himachal Pradesh 177212, India \\ *Corresponding author: math.gurdeep@gmail.com
}

Received January 11, 2019; Revised February 28, 2019; Accepted April 04, 2019

\begin{abstract}
This Paper deals with the Stability of a micropolar fluid layer heated from below in the presence of uniform magnetic field. The basic hydrodynamic equations of magneto-microploar fluid layer heated from below are modified by using Boussinesq approximation and then the linearized perturbation equations are converted into a characteristic value problem with the help of Normal mode analysis. The expressions for Rayleigh number are obtained by using the variational principle. The effect of magnetic field and micropolar parameter on the Rayleigh numbers are discussed andupper bounds of critical Rayleigh number for all type of boundaries are obtained by using variational method.
\end{abstract}

Keywords: Rayleigh number, principle of exchange of stabilities, Variational principle, micro rotation, Boussinesq approximation, magnetic induction

Cite This Article: Joginder Singh Dhiman, and Gurdeep Singh, "Stability Analysis of a Magneto Micropolar Fluid Layer by Variational Method." American Journal of Applied Mathematics and Statistics, vol. 7, no. 3 (2019): 86-92. doi: 10.12691/ajams-7-3-1.

\section{Introduction}

Thermal convection occurs in nature in so many forms and over such a wide range of scales that it could be claimed with some justification that convection represents the most common fluid flow in the universe.

The experiments by Bénard [1] particular have attracted great attention and are today considered as classical in fluid mechanics. The Bénard stability problem was first formulated and mathematically solved by Loard Rayleigh [2] for the case of free boundaries with a linear temperature gradient. A comprehensive account of the linearized stability theory of Rayleigh-Bénard convection problem in the presence of uniform rotation has been given in chanderasekhar [3].

Some years ago, Eringen [4] developed the theory of micropolar fluids in which local effects arising from micro-Structure and intrinsic motions of the fluid elements are taken into account. Ahmadi [5] studies the stability of a layer of micropolar fluid heated from below using linear theory as well as energy method the derived lower bounds for critical Rayleigh number. The profiles on Benard convection in micropoler fluids is also discussed by Narasimha, MY [6]. Dattav and Sastry [7] also discussed the theory of micropolar fluid layer heated from below and obtained exact solution of the eigen value problem. Joginder Singh Dhiman, Praveenkumar Sharma and Gurdeep Singh.

[8] studied the stability of micropolar fluid layer heated from below by variational principle.
The stability of magneto-micropolar fluid motion was studied by G. Ahmadi and M. Shahinpoor [9]. The stability method employed by G. Ahmadi and M. Shahinpoor was an energy technique due to James Serrin [10].

Our aim in this paper is to verify the stability of magneto-micropolar fluid motion by using the variational method and we have established this by finding the upper bounds for critical Rayleigh numbers for all combinations of different type of boundaries.

\section{Mathematical Analysis}

\subsection{Physical Problem}

A viscous finitely heat and electrically conducting, micropolar fluid is statically confined between two horizontal boundaries $Z=0$ and $Z=d$ of infinite extension and finite vertical depth which are maintained at uniform temperatures $T_{0}$ and $T_{1}\left(T_{0}>T_{1}\right)$ respectively in the presence of uniform magnetic field acting antiparallel to the force field of gravity.

\subsection{Hydrodynamic Equations and Boundary Conditions}

The basic thermodynamic equations of the problem of thermal stability of magnetic micropolar fluid layer heated from below are modified by using the usual steps of Boussinesq of approximation and Normal mode. Analysis the non dimensional linearized perturbation equations with boundary conditions are as follows: 


$$
\begin{gathered}
\left(D^{2}-a^{2}\right)\left[D^{2}-a^{2}-\frac{p}{\sigma(1+K)}\right] w \\
=-\frac{K}{1+K}\left(D^{2}-a^{2}\right) G+\frac{R a^{2} \theta}{1+K}-\frac{Q}{1+K}\left(D^{2}-a^{2}\right) h_{z} \\
\left(D^{2}-a^{2}-p\right) \theta=-w \\
\left(D^{2}-a^{2}-\frac{n_{1} p+2 k \sigma}{\sigma C_{o}}\right) G=\frac{K}{C_{0}}\left(D^{2}-a^{2}\right) W \\
\left(D^{2}-a^{2}-\frac{p \sigma_{1}}{\sigma}\right) h_{z}=-D w
\end{gathered}
$$

The boundary conditions are

$$
w=0=q=G=D W \text { at } z=0, z=1 .
$$

(When both the boundaries are rigid).

$$
w=0=q=G=D^{2} w \text { at } z=0, z=1
$$

(When both boundaries are free) and

$$
h_{z}=0 \text { at } z=0, z=1
$$

(When both boundaries are either rigid or free, but boundaries are conducting).

In the foregoing equations;

$$
K=\frac{k}{\mu}, n_{1}=\frac{J}{d^{2}}, C_{0}=\frac{\gamma}{\mu d^{2}}
$$

$k$ is dynamic micro rotation viscosity, $\mu$ is the dynamic Newtonain viscosity, $p$ is hydrostatic pressure, $\mathrm{J}$ is microinertia, $\gamma$ is constant stands for coefficient of angular viscosity.

$$
D\left(\equiv \frac{d}{d z}\right) \text { is the differentiation with respect to } z, z \text { in the }
$$

real independent variable, $a^{2}$ is the square of wave number, $\sigma$ is the thermal Prandtl number, $R=\frac{g \alpha \beta \alpha^{4}}{k_{0} \mu}$, $\left(K_{0}=\frac{k^{1}}{P_{0} w}\right)$ is the heat diffusivity is the Rayleigh number, $g$ is the gravitational Acceleration, $\alpha$ is the coefficient of tharmal gradient, $d$ is the depth of layer, $k^{1}$ is the thermometric conductivity, $v$ is the kinematic $\mu$ is viscosity, $w, \theta$ and $\mathrm{G}$ are the pertubations in vertical velocity, temperature and microrotation respectively and $Q=\frac{\mu_{e} H^{2} d^{2}}{4 \pi P_{o} \gamma \eta}$.

The system of equations (1) - (4) together with boundary condition (5) - (7) constitutes an eigen value problem for $\mathrm{R}$ for given values of other parameters $\sigma, c_{0}, n_{1}, a^{2} \mathrm{~K}$ and $\mathrm{Q}$.

\subsection{Principle of Exchange of Stabilities (PES)}

Firstly in the present problem, we shall see that whether the PES is valid or not for magneto micropolar fluid heated from below.
So multiplying equation (1) by $w^{*}$ (the complex conjugate of $w$ ) and integrating over the range $0 \leq z \leq 1$, a suitable number of times by using relevant boundary conditions (5)-(7) we get

$$
\begin{aligned}
& \int_{.0}^{1}\left\{\left|D^{2} W\right|^{2}+a^{2}|w|^{2}+2 a^{2}|D w|^{2}\right\} d z \\
& \left.+\frac{p}{\sigma(1+k)} \int_{0}^{1}\{|D w|\}^{2}+a^{2}|w|^{2}\right\} d z \\
& =\frac{-K}{k+1} \int_{0}^{1} w^{*}\left(D^{2}-a^{2}\right) G d z+\frac{a^{2} R}{1+k} \int_{0}^{1} w^{k} \theta d z \\
& -\frac{Q}{1+K} \int_{0}^{1} W^{*}\left(D^{2}-a^{2}\right) D h_{z} .
\end{aligned}
$$

Taking the complex conjugate of equation (3) and multiplying the resulting equation by $\mathrm{G}$ on both sides and integrating it over the range of $z$, a suitable number of times by using boundary conditions (5) - (7), we get

$$
\begin{aligned}
& \int_{0}^{1}\left\{|D G|^{2}+a^{2}|G|^{2}+\frac{n_{1} p^{*}+2 k \sigma}{\sigma c_{0}}|G|^{2}\right\} d z \\
& =-\frac{K}{C_{0}} \int_{0}^{1} W^{*}\left(D^{2}-a^{2}\right) G d z
\end{aligned}
$$

By using equation (9) is equation (8) and integrating by parts the resulting equation, we get

$$
\begin{aligned}
& \int_{0}^{1}\left\{\left|D^{2} w\right|^{2}+a^{4}|w|^{2}+2 a^{2}|D w|^{2}\right\} d z \\
& +\frac{p}{\sigma(1+K)} \int_{0}^{1}\left\{\left|D^{2} w\right|^{2}+a^{2}|w|^{2}\right\} d_{z} \\
& \left.=\frac{C_{0}}{1+k} \int_{0}^{1}\left\{|D G|^{2}+a^{2}|G|^{2}\right\}+\frac{n_{1} p^{*}+2 k \sigma}{\sigma C_{0}}|G|^{2}\right\} d z \\
& +\frac{R a^{2}}{1+k} \int_{0}^{1}\left\{|D \theta|^{2}+a^{2}|\theta|^{2}+p^{*}|\theta|^{2}\right\} d z \\
& +\frac{Q}{1+k}\left[2 a^{2} \Gamma+\int_{0}^{1}\left|D^{2} h_{z}\right|^{2}+a^{2}\left|D h_{z}\right|^{2} a^{4}\left|h_{z}\right|^{2}\right] d_{z} \\
& \quad-\frac{Q p * \sigma_{1}}{\sigma(1+k)}\left[\Gamma+\int_{0}^{1}\left\{\left|D h_{z}\right|^{2}+a^{2}\left|h_{z}\right|^{2}\right\} d z\right]
\end{aligned}
$$

Where $\Gamma=a\left\{\left(\left|D h_{z}\right|^{2}\right)_{0}+\left(\left|h_{z}\right|^{2}\right)_{1}\right\} \geq 0$.

Now equating real and imaginary parts of both sides of equation (10) and canceling $\mathrm{p}_{i}(\neq 0)$ (supposition) throughout from imaginary part, we get;

$$
\begin{aligned}
& \frac{1}{\sigma(1+k)} \int_{0}^{1}\left(|D W|^{2}+a^{2}|w|^{2}\right) d z+\frac{C_{0}}{1+K} \int_{0}^{1} \frac{n_{1}}{\sigma C_{0}}|G|^{2} d z \\
& +\frac{R a^{2}}{1+K} \int_{0}^{1}|\theta|^{2} d z=\frac{Q \sigma_{1}}{\sigma(1+k)}\left[\Gamma+\int_{0}^{1}\left|D h_{z}\right|^{2}+a^{2}\left|h_{z}\right|^{2} d z\right] .
\end{aligned}
$$


Now, multiplying the equation (4) by $h_{z}^{*}$ (the complex conjugate of $h_{z}$ ) and integrating the resulting equation over the vertical range of $z$, a suitable number of times and making use of either boundary conditions (5) - (7), we have.

$$
\begin{aligned}
& \Gamma+\int_{0}^{1}\left(\left|D h_{z}\right|^{2}+a^{2}\left|h_{z}\right|^{2}\right) d z+\frac{p \sigma_{1}}{\sigma} \int_{0}^{1}\left|h_{z}\right|^{2} d z \\
& =-\int_{0}^{1} w D h_{z}^{*} d z
\end{aligned}
$$

Equating real parts from both sides of above equation, we have:

$$
\begin{aligned}
& \Gamma+\int_{0}^{1}\left(\left|D h_{z}\right|^{2}+a^{2}\left|h_{z}\right|^{2}\right) d z+\frac{p_{r} \sigma_{1}}{\sigma} \int_{0}^{1}\left|h_{z}\right|^{2} d z \\
& =\text { Real part of }\left(-\int_{0}^{1} w D h_{z}^{*} d z\right) \\
& \leq\left|-\int_{0}^{1} W D h_{z}^{*} d z\right| \leq \int_{0}^{1}|W|\left|D h_{z}\right| d z \\
& \leq \frac{1}{2}\left[\left\{\int_{0}^{1}|w|^{2} d z\right\}+\left\{\int_{0}^{1}|D h z|^{2} d z\right\}\right] .
\end{aligned}
$$

Since $p_{r} \geq 0$ from above inequality, we have

$$
\Gamma+\int_{0}^{1}\left(\left|D h_{z}\right|^{2}+a^{2}\left|h_{z}\right|^{2}\right) d z<\int_{0}^{1}|w|^{2} d z-a^{2} \int_{0}^{1}\left|h_{z}\right|^{2} d z \text { (14) }
$$

Further, since $w(0)=0=w(1)$, we have the Reyleigh - Ritz inequality namely;

$$
\int_{0}^{1}|w|^{2} d z \leq \frac{1}{\Pi^{2}} \int_{0}^{1}|D w|^{2} d z
$$

Using inequalities (14) and (15) is equation (11), we get

$$
\begin{aligned}
& {\left[\frac{\Pi^{2}}{\sigma(1+K)}-\frac{Q \sigma_{1}}{\sigma(1+k)}\right] \int_{0}^{1}|w|^{2} d z+\frac{Q \sigma_{1} a^{2}}{\sigma(1+k)} \int_{0}^{1}\left|h_{z}\right|^{2}} \\
& +\frac{R a^{2}}{1+k} \int_{0}^{1}|\theta|^{2} d z+\frac{n_{1}}{\sigma(1+k)} \int_{0}^{1}|G|^{2} d z \\
& <0 .
\end{aligned}
$$

It clearly follows from above inequality that

$$
\begin{gathered}
\frac{\Pi^{2}}{\sigma(1+K)}-\frac{Q \sigma_{1}}{\sigma(1+K)}<0 \\
Q>\frac{\Pi^{2}}{\sigma_{1}},
\end{gathered}
$$

Which is a contradiction to our suppositon that $\mathrm{p}_{i} \neq 0$. Hence, we must have $\mathrm{p}_{i}=0$, In particular $\mathrm{p}_{r}=0 \Rightarrow \mathrm{p}_{i}=0$, hence PES is Valid.

Therefore, we have the result that PES is not valid or have over stability when $Q \sigma_{1}>\Pi^{2}$, which can also be seen in classical magneto hydrodynamic Bénard convection.

\subsection{Variational Principle}

When instability sets is as stationary convection, the marginal state will be characterized by $\mathrm{p}=0$ and basic equations (1) - (4) reduce to

$$
\begin{gathered}
\left(D^{2}-a^{2}\right)^{2} w=\frac{K}{1+K}\left(D^{2}-a^{2}\right) G+\frac{1}{1+K} a^{2} R \theta \\
\quad-\frac{Q}{1+K} D\left(D^{2}-a^{2}\right) h_{z} \\
\left(D^{2}-a^{2}\right) \theta=-w \\
\left(D^{2}-a^{2}-\frac{2 K}{C_{0}}\right) G=\frac{K}{C_{0}}\left(D^{2}-a^{2}\right) w \\
\left(D^{2}-a^{2}\right) h_{z}=-D w .
\end{gathered}
$$

From equation (17), letting

$$
\begin{aligned}
F= & \left(D^{2}-a^{2}\right)^{2} w+\frac{K}{1+K}\left(D^{2}-a^{2}\right) G \\
& +\frac{Q}{1+K} D\left(D^{2}-a^{2}\right) h_{z}
\end{aligned}
$$

implies,

$$
F=\frac{1}{1+K} a^{2} R \theta .
$$

Operating the above equation lay $\left(D^{2}-a^{2}\right)$ and using equation (18), we get:

$$
\left(D^{2}-a^{2}\right) \mathrm{F}=-\frac{a^{2}}{1+K} R w .
$$

Multiplying the above equation by $\mathrm{F}$ and integrating the resulting equation over the range of $z$, we have

$$
\int_{0}^{1} F\left(D^{2}-a^{2}\right) F d z=-\frac{a^{2} R}{1+K} \int_{0}^{1} w F d z
$$

Since $F(0)=0=F(1)$ because of equation (22), therefore, we have

$$
\int_{0}^{1} F\left(D^{2}-a^{2}\right) F d z=-\int_{0}^{1}\left((D F)^{2}+a^{2} F^{2}\right) d z
$$

Now integrating the equation (24) by using $\mathrm{F}$ from equation (22) and integrating a suitable number of times by using relevant boundary conditions, we get:

$$
\frac{K}{1+K} \int_{0}^{1} w\left(D^{2}-a^{2}\right) G d z=\frac{K}{1+K}\left\{\int_{0}^{1} G\left(D^{2}-a^{2}\right) d z\right\} .
$$

By using equation (19) in above equation and integrating the resulting equation by parts a suitable number of times and using boundary conditions on $\mathrm{G}$, we get

$$
\begin{aligned}
& \frac{k}{1+k} \int_{0}^{1} w\left(D^{2}-a^{2}\right) G d z \\
& =\frac{-C_{0}}{1+k}\left\{(D G)^{2}+\left(a^{2}+\frac{2 k}{C_{0}}\right) G^{2}\right\} d z .
\end{aligned}
$$


Now, by using all these equations (25), (27), in the equation (24), we have :

$$
\begin{aligned}
& \int_{0}^{1}\left((D F)^{2}+a^{2} F^{2}\right) d z \\
& =\frac{a^{2} R}{1+K}\left[\int_{0}^{1}\left[\left(D^{2}-a^{2}\right)\right]^{2}\right] d z \\
& -\frac{C_{0}}{1+K} \int_{0}^{1}\left\{(D G)^{2}+\left(a^{2}+\frac{2 K}{C_{0}}\right) G^{2}\right\} d z \\
& +\frac{Q}{1+K} \int_{0}^{1}(D W)^{2} d z
\end{aligned}
$$

Or

$$
R=\frac{(K+1) \int_{0}^{1}\left[(D F)^{2}+a^{2} F^{2}\right] d z}{a^{2}\left\{-\frac{C_{0}}{1+K} \int_{0}^{1}\left\{(D G)^{2}+\left(a^{2}+\frac{2 K}{C_{0}}\right)\right\} G^{2}\right\}}
$$

which may be written as

$$
R=\frac{(K+1) I_{1}}{a^{2} I_{2}}
$$

Where

$$
I_{1}=\int_{0}^{1}\left\{|D F|^{2}+a^{2} F^{2}\right\} d z
$$

And

$$
\begin{aligned}
I_{2}= & \int_{0}^{1}\left[\left(D^{2}-a^{2}\right) w\right]^{2} d z-\frac{C_{0}}{1+K} \int_{0}^{1}(D G)^{2} \\
& \left.+\left(a^{2}+\frac{2 K}{C_{0}}\right) G^{2}\right\} d z+\frac{Q}{1+K} \int_{0}^{1}(D w)^{2} d z .
\end{aligned}
$$

\subsection{Stationary Property}

The stationary property is R given by equation (28) is checkedjust by giving a small variation $\delta \mathrm{W}$ to $\mathrm{w}, \delta \mathrm{G}$ to $\mathrm{G}$ and $\delta h_{z}$ to $h_{z}$, which are again compatible with boundary conditions i.e.

$d G=0, d w=0, d h z=0$ and $d F=0$ for $z=0$ and $z=1$.

This can be easily checked that $\delta \mathrm{R}=0$ if $\left(\mathrm{D}^{2}-a^{2}\right) \mathrm{F}=$ $\frac{-R a^{2} w}{K+1}$ and conversely if $\delta \mathrm{R}=0$ for any arbitrary Variation, then $\left(D^{2}-a^{2}\right) \mathrm{F}=\frac{R a^{2}}{K+1} w$.

\subsection{Minimum Property}

Now, we shall show that the lowest characteristic value of $\mathrm{R}$ is indeed, a true minimum.

Let $\mathrm{R}_{\mathrm{i}}$ be a characteristic value and let corresponding characteristic function be distinguished by a subscript $\mathrm{i}$. Then from equation (23), we have

$$
\left(D^{2}-a^{2}\right) F i=\frac{-R_{i} a^{2}}{K+1} w_{i}
$$

and

$$
\mathrm{W}_{\mathrm{i}}=\mathrm{F}_{\mathrm{i}}=0 \text { at } z=0 \text { and } z=1
$$

and either

$$
D^{2} \delta w_{i}=0 \text { or } D^{2} \delta w_{i}=0 \text { at } z=0 \text { and } z=1 .
$$

Multiplying equation (29) by $F_{j}$ and integrating the resulting equation over vertical range of $\mathrm{z}$, we have

$$
\int_{0}^{1} F_{j}\left(D^{2}-a^{2}\right) F_{i} d z=-\frac{R_{i} a^{2}}{1+K} \int_{0}^{1} F_{j} W_{i} d z .
$$

By using equation (21), we have

$$
\begin{aligned}
& \int_{0}^{1} F_{j}\left(D^{2}-a^{2}\right) F_{i} d_{z} \\
& =-\frac{R_{i} a}{1+K} \int_{0}^{1} W_{j}\left\{\begin{array}{l}
\left.\left(D^{2}-a^{2}\right) W_{g}+\frac{K}{1+K}\left(D^{2}-a^{2}\right) G_{j}\right\} \\
+\frac{Q}{1+K}\left(D^{2}-a^{2}\right) D h_{z}
\end{array}\right\} d Z \\
& \int_{0}^{1} F_{j}\left(D^{2}-a^{2}\right) F_{i} d_{z} \\
& =-\frac{R_{i} a}{1+K} \int_{0}^{1} W_{i}\left\{\begin{array}{l}
\left(D^{2}-a^{2}\right) W_{j}+\frac{K}{1+K}\left(D^{2}-a^{2}\right) G_{j} \\
+\frac{Q}{1+K}\left(D^{2}-a^{2}\right) D h_{z}
\end{array}\right\} d Z
\end{aligned}
$$

Integrating the integrants on right hand side of above equation by parts, a suitable number of times with the help of boundary conditions(30) - (31), We have

$$
\int_{0}^{1} W_{i} F_{j} d z=\int_{0}^{1} W_{j} F_{i} d z
$$

Now, $\quad \int_{0}^{1} F_{j}\left(D^{2}-a^{2}\right) F_{i} d z=\int_{0}^{1}\left(D F_{j} D F_{i}+a^{2} F_{j} F_{i}\right) d z$ using this equation in (29) and interchanging $i$ and $j$ then subtracting the resulting equations, we have

$$
\left(R_{i}-R_{j}\right) \frac{a^{2}}{1+K} \int_{0}^{1} W_{i} F_{j} d z=0
$$

Since, $\mathrm{R}_{i} \neq \mathrm{R}_{j}$ we have;

$$
\begin{array}{r}
\int_{0}^{1} W_{i} F_{j} d_{z}=0 \text { if } i \neq j \\
\text { if } i=j .
\end{array}
$$


The functions $\mathrm{W}_{\mathrm{i}}$ form an orthogonal set.

Let

$$
W=\sum_{i=1}^{\infty} A_{i} W_{i}, \text { Where } A_{i}=\int_{0}^{1} W F_{i} d z
$$

Similarly using the expanded forms $W_{i}$ terms of basic set functions for $\mathrm{G}, \mathrm{F}$, and $h_{z}$, the values of integrals $\mathrm{I}_{1}$ and $\mathrm{I}_{2}$ and equation (28) having the following values,

$$
\begin{array}{r}
\mathrm{I}_{1}=\frac{a^{2}}{1+k} \sum_{i=1}^{\infty} A i^{2} R i(\text { for } i=j) \\
\text { also, } \mathrm{I}_{2}=\int_{0}^{1} W F d z=\int_{0}^{1} \sum_{i=1}^{\infty} A_{i} w_{i} \sum_{j=1}^{\infty} A_{j} F_{j} d z \\
I_{2}=\sum_{i=1}^{\infty} A i^{2}(\text { for } i=j)
\end{array}
$$

Using these values of $I_{1}$ and $I_{2}$ is equations (28), we have

$$
\begin{array}{r}
R=\frac{(1+K) \frac{a^{2}}{1+k} \sum_{i=1}^{\infty} A_{i}^{2} R_{i}}{a^{2} \sum_{i=1}^{\infty} A_{i}^{2}} \\
R=\frac{\sum_{i=1}^{\infty} A_{i}^{2} R_{i}}{\sum_{i=1}^{\infty} A_{i}^{2}}=\sum_{i=1}^{\infty} R_{i} B_{i}{ }^{2}
\end{array}
$$

When $\sum_{i=1}^{\infty} \mathrm{B}_{i}^{2}=1$.

In follows from equation (34) that

$$
(\mathrm{R}-\mathrm{R})=\sum_{i=1}^{\infty}\left(R_{i}-R_{c}\right) R_{i}^{2} \geq 0 .
$$

Since $R_{i} \geq R_{c} \forall i$.

Thus, we have

$$
R_{c} \leq R=\frac{(1+K) I_{1}}{a^{2} I_{2}}
$$

This shows that the quantity on R.H.S has a true minimum.

\subsection{Upper Bounds for Critical Raylight \\ Number for All Combination of Boundary Conditions}

Let

$$
F=\operatorname{Cos} \prod z
$$

Which obviously satisfies the boundary conditions

$$
\mathrm{F}=0 \text { at } \mathrm{z}=-\frac{1}{2} \text { and } z=+\frac{1}{2},
$$

where the origin has been shifted to midway for convenience in computations.

Using the value of $\mathrm{F}$ from equation (36) in equation (21), we obtain

$$
\begin{aligned}
& \left(D^{2}-a^{2}\right) W+\frac{K}{1+K}\left(D^{2}-a^{2}\right) G \\
& +\frac{Q}{1+k}\left(D^{2}-a^{2}\right) D h_{z}=\operatorname{Cos} \Pi z
\end{aligned}
$$

Operating the above equation by $\left(D^{2}-a^{2}-\frac{2 K}{C_{o}}\right)$ and using the equations (19) - (20), We get

$$
\begin{aligned}
- & \left\{\Pi^{2}+\left(a^{2}+\frac{2 K}{C_{o}}\right)\right\} \operatorname{Cos} \pi Z \\
= & \left(D^{2}-a^{2}-\frac{2 K}{C_{0}}\right)\left(D^{2}-a^{2}\right)^{2} w \\
& +\frac{K^{2}}{C_{o}(1+K)}\left(D^{2}-a^{2}\right)^{2} w \\
& -\frac{Q}{1+K} D^{2}\left(D^{2}-a^{2}-\frac{2 K}{C_{0}}\right) w
\end{aligned}
$$

The general solution of above differential equation is given by

$$
\begin{aligned}
W= & B_{1} \operatorname{Cosh} x_{1} z+B_{2} \operatorname{Cosh} x_{2} z \\
& +B_{3} \operatorname{Cosh} x_{3} z+A \operatorname{Cos} \prod z
\end{aligned}
$$

Where $x_{1}^{2}, x_{2}^{2}, x_{3}^{2}$ are roots of auxiliary equation of (38) and

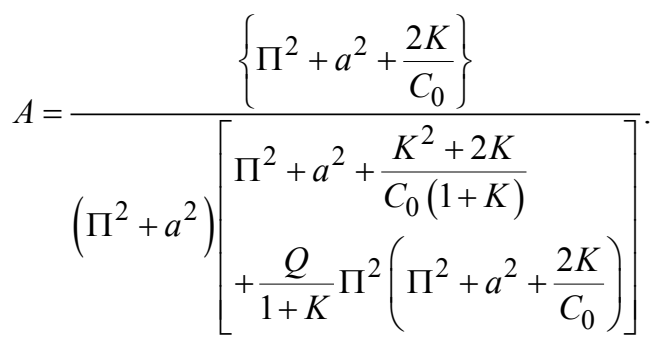

Case I: when both the boundaries are dynamically free. By using equation (39), we have

$$
\begin{aligned}
D w= & B_{1} x_{1}, \sinh x_{1} z+B_{2} x_{2} \sinh x_{2} z \\
& +B_{3} x_{3} \sinh x_{3} z-\prod A \sin \prod z \\
D^{2} w= & B_{1} x_{1}^{2} \operatorname{Cosh} x_{1} z+B_{2} x_{2}^{2} \operatorname{Cosh} x_{2} z \\
& +\cosh x_{3} z-\pi 2 A \cos \prod z
\end{aligned}
$$

And

$$
\theta=-\left\{\begin{array}{l}
\frac{B_{1}}{x_{1}^{2}-a^{2}} \operatorname{Cos} h x_{1} z+\frac{B_{2}}{x_{2}^{2}-a^{2}} \operatorname{Cos} h x_{2} z \\
+\frac{B_{3}}{x_{3}^{2} z-a^{2}} \operatorname{Cos} h x_{3} z-\frac{A \cos \Pi z}{\Pi^{2}+a^{2}}
\end{array}\right\}
$$

Since, both the boundaries are dynamically free, so boundary conditions are $\mathrm{W}=0 \mathrm{D}^{2} w$ at $z= \pm \frac{1}{2}$ and $\theta=0$ at $z= \pm \frac{1}{2}$. 
By using these boundary conditions is above equations, we have

$$
\mathrm{B}_{1}=\mathrm{B}_{2}=\mathrm{B}_{3}=0 \text {. }
$$

From equation (39), we get $W=A \cos \prod z$, when $F=\operatorname{Cos} \prod z$.

Now, evaluating $I_{1}$ and $I_{2}$ is equation (28), we have

$$
\mathrm{I}_{1}=I_{1}=\int_{-1 / 2}^{1 / 2}\left\{(D F)^{2}+a^{2} F^{2}\right\} d z=\frac{\pi^{2}+a^{2}}{2}
$$

and $\mathrm{I}_{2}=\int_{-1 / 2}^{1 / 2} w F d z=A / 2$.

Using these values of $I_{1}$ and $I_{2}$ in equations (35), we get $R_{c} \leq \frac{(k+1)\left(\Pi^{2}+a^{2}\right)}{a^{2} A}$

Now, using the value of $\mathrm{A}$, we have

$$
R_{C} \leq \frac{(K+1)\left(\Pi^{2}+a^{2}\right)\left\{\begin{array}{l}
\left(\Pi^{2}+a^{2}\right)\left(\Pi^{2}+a^{2}+\frac{2 K}{C_{0}}\right) \\
-\frac{K^{2}}{C_{0}(1+K)}\left(\Pi^{2}+a^{2}\right)^{2} \\
+\frac{Q \Pi^{2}}{1+K}\left(\Pi^{2}+a^{2}+\frac{2 K}{C_{0}}\right)
\end{array}\right\}}{a^{2}\left(\Pi^{2}+a^{2}+\frac{2 K}{C_{0}}\right)}
$$

letting $\mathrm{k} \rightarrow \infty$ in the above inequality, we have :

$$
\operatorname{Lt.}_{k \rightarrow \infty} \frac{R_{c}}{K} \leq \frac{\left(\pi^{2}+a^{2}\right)^{3}}{2 a^{2}}
$$

Which is the upper bound for $\mathrm{R}_{\mathrm{c}}$ for free-free boundaries,

\section{Case II One Rigid - One Free Boundary:}

Let us take lower boundary as rigid and upper dynamically free, so using these boundary conditions in the equations (39) - (41) we get:

$$
\begin{gathered}
B_{1} \operatorname{Cosh}\left(\frac{x_{1}}{2}\right)+B_{2} \operatorname{Cos} h\left(\frac{x_{2}}{2}\right)+B_{3} \operatorname{Cos} h\left(\frac{x_{3}}{2}\right)=0 \\
x_{1} B_{1} \operatorname{Sin} h\left(\frac{x_{1}}{2}\right)+x_{2} B_{2} \operatorname{Sin} h\left(\frac{x_{2}}{2}\right)+B_{3} x_{3} \operatorname{Sin} h\left(\frac{x_{3}}{2}\right) \\
=\Pi A \\
\quad x_{1}^{2} B_{1} \operatorname{Cos} h\left(\frac{x_{1}}{2}\right)+x_{2} B_{2} \operatorname{Cos} h\left(\frac{x_{2}}{2}\right) \\
\quad+B_{3} x_{3} \operatorname{Cos} h\left(\frac{x_{3}}{2}\right)=0
\end{gathered}
$$

On solvingthe above equations, we have :

$$
B_{1}=\frac{\Pi A}{\Delta} \operatorname{Cos} h\left(\frac{x_{2}}{2}\right) \operatorname{Cos} h\left(\frac{x_{3}}{3}\right)\left\{x_{3}^{2}-x_{2}^{2}\right\}
$$

$$
\begin{aligned}
& B_{2}=\frac{\Pi A}{\Delta} \operatorname{Cosh}\left(\frac{x_{1}}{2}\right) \operatorname{Cosh}\left(\frac{x_{3}}{2}\right)\left\{x_{3}^{2}-x_{1}^{2}\right\} \\
& B_{3}=\frac{\Pi A}{\Delta} \operatorname{Cosh}\left(\frac{x_{1}}{2}\right) \operatorname{Cosh}\left(\frac{x_{2}}{2}\right)\left\{x_{3}^{2}-x_{1}^{2}\right\}
\end{aligned}
$$

Where

$$
\begin{aligned}
\Delta= & \operatorname{Cosh}\left(\frac{x_{1}}{2}\right) \operatorname{Cos} h\left(\frac{x_{2}}{2}\right) \operatorname{Cosh}\left(\frac{x 3}{2}\right)\left(\frac{x_{2}}{2}\right) \\
& \times\left\{\begin{array}{l}
\left.x_{1} \tan h\left(\frac{x_{1}}{2}\right)-x_{2} \tan h\left(\frac{x_{2}}{2}\right)\right)\left(x_{2}^{2}-x_{3}^{2}\right) \\
\left.-\left(x_{1}^{2}-x_{2}^{2}\right)\left(x_{2} \tan h\left(\frac{x_{2}}{2}\right)-x_{3} \tan h\left(\frac{x 3}{2}\right)\right)\right\} .
\end{array}\right.
\end{aligned}
$$

Now using the values of $\mathrm{W}$ and $\mathrm{F}$ is integrals $\mathrm{I}_{1} \& 1_{2}$, we have

$$
\begin{gathered}
I_{1}=\frac{\Pi^{2}+a^{2}}{2} \text { and } \\
I_{2}=\frac{2 \Pi B_{1} \operatorname{Cos} h\left(\frac{x_{1}}{2}\right)}{x_{1}^{2}+\Pi^{2}}+\frac{2 \Pi B_{2} \operatorname{Cosh}\left(\frac{x_{2}}{2}\right)}{x_{2}^{2}+\Pi^{2}} \\
+\frac{2 n B_{3} \operatorname{Cosh}\left(\frac{x_{3}}{2}\right)}{x_{3}^{2}+\Pi^{2}}+\frac{\mathrm{A}}{2}
\end{gathered}
$$

By using these values of $I_{1} \& I_{2}$ is inequality (35), We have

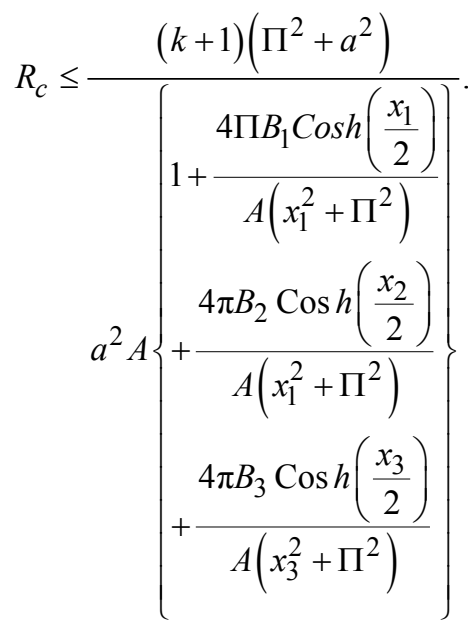

Now, by using the values of $\mathrm{B}_{1}, \mathrm{~B}_{2}, \mathrm{~B}_{3}, \Delta \mathrm{A}$ and letting $\mathrm{K} \rightarrow \infty$ in above inequality, we have

$$
\underset{k \rightarrow \infty}{\text { Lt. }} \frac{R_{c}}{K} \leq \frac{\left(\pi^{2}+a^{2}\right)^{3}}{2 a^{2}}
$$

Which is upper bound for $\mathrm{R}$, for one rigid-one free boundaries.

\section{Case III. For both rigid-rigid boundaries:}

Now by using equations (39), (40) and (42), with boundary conditions in $\mathrm{w}, \mathrm{Dw}$ and $\theta$ (proceeding same as in the previous two cases), We have: 


$$
\operatorname{Lt}_{K \rightarrow \infty} \frac{R_{c}}{K} \leq \frac{\left(\pi^{2}+a^{2}\right)^{3}}{2 a^{2}}
$$

Which is upper bound for $\mathrm{R}_{\mathrm{c}}$ for both rigid-rigid boundaries.

\section{Conclusion}

In this paper it is verified that PES is valid for magneto-micropolar fluid motion for all type of boundary conditions when heated from below. There is over stability only when $Q \sigma_{1}>\Pi^{2}$, which can also be seen in classical magneto hydrodynamic Bénard convection. The upper bounds for Critical Rayleigh number, that is $L_{K \rightarrow \infty} \frac{R_{c}}{K} \leq \frac{\left(\Pi^{2}+a^{2}\right)^{3}}{2 a^{2}}$ are obtained by using variational principle having same value for all type of boundary conditions namely, both free-free, one rigid-one free and both rigid-rigid. Further, these upper bounds depend upon the value of micro rotation coefficient $\mathrm{K}$ in magneto-micropolar fluid heated from below. The inequality for upper bounds clearly shows that the increasing value of $\mathrm{K}$ increases the Rayleigh number $\mathrm{R}$.
Thus K plays a stabilizing role in the system.

\section{References}

[1] Benard, H.; Les tourbillons cellulairs dons une nappe liquids; Revue, generade des sciences pures et Applications, 1900: 11, $1261 \& 1309$.

[2] Lord Rayleigh: On the connective currents in a horiyontal layer of fluid when higher temperature is one the upper side; phil.plag., 1990, 32, 529.

[3] Chandrasekher S.. Hydrodynamic and Hydromagnetic stability, Oxford University Press, Aman House, London, 1961.

[4] Eringen, A.C.: 'Theory of Micropolar Bluids; J. Math. Mech. 1906, $16,1,1-18$.

[5] Ahmadi, G.: Stability of a micropolar fluid Layer Heated from Below', Int. J. Engng. Sci., 1976, 14, 81-89.

[6] Narasimha, M.Y.: 'Analysis of non uniform temp/ profiles on Benard convection in micropolar fluids; App. Math. comput, 2003: 134, 473-486.

[7] Datta, A. B. and Sastry, V.U.K. 'Thermal Notability of a Horigontal Layer of, Micropolar fluid Heated from Bolows, Int. J. Engng. Sci. 1976:14, 631-637.

[8] Joginder S. Dhiman, Praveen K. Sharma and Gurdeep Singh: 'Convective stability analysis of a micropolar fluid layer by variational method' The Chinese Society of Theoretical and Applied Machanics Letters; 2011.

[9] Ahmadi, G. and Shahinpoor, M.; 'Universal stability of magneto-micropolar fluid motions; Int. J. Engn. Sci, 1947, 12, 657-663.

[10] J.S. ERRIN, Arch, ration. Mech. Analysis 1950, $3,1$. 